\title{
ANNUAL INDEX 1994
}

\section{AUTHOR INDEX}

Beaver, K.A., Mann, W.C.: Provider Skills for Delivering Computer Access Services: An Assistive Technology Team Approach, 109

Berent, G.P.: See Kelly, R.R.., 26

Biggie, L.: The Second National Search for Computer Applications to Assist Persons with Disabilities: Entries Related to Hearing, Speech, and Language, 11

Boone, R.: See Higgins, K., 137

Bowe, F.G.: Technologies That Hear and Talk, 1

Brabyn, J.: See Crandall, W.F., 203

Brabyn, J.: Rehabilitation Engineering Research in Blindness, Visual Impairment and Multisensory Loss, 266

Brandt, R.P.: ITU-T Recommended V.18: The First Communications Standard for the Deaf, 199

Brusnighan, D.A., Ploss, A.J., Getts, M.L.: Farming with a Hearing Impairment, 39

Buckley, G.J.: Technologies for People with a Hearing Loss: New Initiatives in Postsecondary Education, 69

Carey, D.M., Sale, P.: Practical Considerations in the Use of Technology to Facilitate the Inclusion of Students with Severe Disabilities, 77

Clark, A.: See Weisman, G., 258

Constantino, S.Z.: See Mistrett, S.G., 148

Corley, P.: See Zorfass, J.M., 158

Crandall, W.F., Gerrey, W., Brabyn, J.: Remote Reading for Visually Impaired People: A New Application for Telecommunications, 203

Dowler, D.L., Hirsh, A.: Accommodations in the Workplace for People Who are Deaf or Hard of Hearing, 15

Ethier, D.: See Zorfass, J.M., 158

Fernie, G., Maki, B.: Research and Development in Assistive Technology at the Centre for Studies in Aging in Toronto, 315
Fischer, S.: See Kelly, R.R., 26

Fitzgerald, G.E.: Using the Computer with Students with Emotional and Behavioral Disorders, 87

Foulds, R.: The Applied Science and Engineering Laboratories: The University of Delaware and the A.I. duPont Institute, 291

Frisina, D.R.: See Scherer, M.J., 62

Fullmer, S.L., Walls, R.T.: Exploring the Internet: The Rehabilitation Community's Access to the Growing Communication Network, 235

Gabella, M.S.: See Kinzer, C.K., 117

Gawley, D.J.: See Gibler, C.D., 218

Gerrey, W.: See Crandall, W.F., 203

Getts, M.L.: See Brusnighan, D.A., 39

Gibler, C.D., Kall, D.A., Kasday, L.R., Gawley, D.J.: The Accessible Design Process: An AT\&T Perspective, 218

Harkins, J.E.: Telecommunications for Deaf and Hard of Hearing Children: Educating the Schools, 195

Harkins, J.E., Levitt, H., Peltz-Strauss, K.: Technology and Telephone Relay Service, 173

Higginbotham, D.J.: See Mann, W.C., 283

Higgins, K., Boone, R.: Site-Based Software: Hypermedia Instructional Applications, 137

Hirsh, A.: See Dowler, D.L., 15

Hurren, M.D.: See Mann, W.C.., 47

Kall, D.A.: See Gibler, C.D., 218

Kasday, L.R.: See Gibler, C.D., 218

Keefe, B.: As Maine Goes ... American Sign Language at a Distance, 72

Kelly, R.R., Samar, V.J., Loeterman, M., Berent, G.P., Parasnis, I., Kirchner, C.J., Fischer, S., Murphy, C.: CC School Project: Personal Captioning Technology Applied to the Language Learning Environment of Deaf Children, 26

Technology and Disability 1994; 3(4):331-334

Copyright $(0) 1994$ by Butterworth-Heinemann. 
Kinzer, C.K., Gabella, M.S., Rieth, H.J.: An Argument for Using Multimedia and Anchored Instruction to Facilitate Mildly Disabled Students' Learning of Literacy and Social Studies, 117

Kirchner, C.J.: See Kelly, R.R., 26

Levitt, H.: See Harkins, J.E., 173

Loeterman, M.: See Kelly, R.R., 26

Lubinski, R: See Mann, W.C., 283

Maki, B.: See Fernie, G., 315

Mann, W.C.: See Beaver, K.A., 109

Mann, W.C., Hurren, M.D., Tomita, M.R.: Assistive Device Needs of Home-Based Elderly Persons with Hearing Impairments, 47

Mann, W.C., Steinfeld, E., Higginbotham, D.J., Lubinski, R., Mullick, A.: The Rehabilitation Engineering Research Center on Aging, 283

McNeal, D.R.: RERC on Technology for Children with Orthopedic Disabilities, 307

Milner, M., Naumann, S.: Rehabilitation Engineering at the Rehabilitation Engineering Department: The Hugh MacMillan Rehabilitation Centre, 321

Mistrett, S.G., Constantino, S.Z., Pomerantz, D.: Using Computers to Increase the Social Interactions of Preschoolers with Disabilities at Community-Based Sites, 148

Mullick, A.: See Mann, W.C., 283

Murphy, C.: See Kelly, R.R., 26

Naumann, S.: See Milner, M., 321

Parasnis, I.: See Kelly, R.R., 26

Peltz-Strauss, K.: See Harkins, J.E., 173

Ploss, A.J.: See Brusnighan, D.A., 39
Pomerantz, D.: See Mistrett, S.G., 148

Ransom, P.: Public Policy/Legislative Trends: Telecommunications Access for People with Disabilities, 165

Remz, A.: See Zorfass, J.M., 158

RESNA: Technology and the Individualized Education Program: A Primer for Parents and Professionals, 100

Reswick, J.B.: What Constitutes Valid Research? Qualitative vs. Quantitative Research, 255

Rieth, H.J.: See Kinzer, C.K., 117

Sale, P.: See Carey, D.M., 77

Samar, V.J.: See Kelly, R.R., 26

Scherer, M.J.: Introduction, Scherer, M.J., Frisina, D.R.: Applying the Matching People with Technologies Model to Individuals With Hearing Loss: What People Say They Want-and Need-from Assistive Technologies, 62

Steinfeld, E.: See Mann, W.C., 283

Tobias, J.: Consumers and Telecommunications, 243

Tobias, J.: Shared Resource Assistive Systems, 213

Tomita, M.R.: See Mann, W.C., 47

Walls, R.T.: See Fullmer, S.L., 235

Weisman, G., Clark, A.: Vermont Rehabilitation Engineering Research Center: Applying Technology to Reduce Low Back Pain, 258

Zorfass, J., Corley, P., Remz, A., Ethier, D.: Promoting Technology in Special Education: Supporting Change Agents, 158

Zorfass, J.M.: Supporting Students with Learning Disabilities: Integrating Technology into an I-Search Unit, 129 


\section{KEYWORD INDEX}

Access, 165, 244

Accessible design, 218

Aging, 47, 283, 315

Agriculture, 39

American Sign Language, 72

Anchored instruction, 117

Assessment, 109

Assistive, 213

Assistive devices, 62, 315

Assistive technology, 100, 109, 258, 283, 291, 307

Augmentative communication, 291

Barriers, 77

Baudot, 199

Behavior, 87

Behavior disorders, 87

Bilingual learning, 26

Blind, 266

Broadband, 1

Bulletin board, 1

Captioning, 1

Change agent, 158

Children with disabilities, 307

Communications, 199

Compatibility, 199

Computer, 72, 77, 87, 109, 148

Computer access, 291, 321

Computer-assisted instruction, 137

Computers in the classrooms, 26

Consumer, 243

Curriculum, 129, 195

Deaf children, 26

Deaf, 11, 72, 173, 195, 199

Deaf-blind, 266

Device evaluation, 62

Disabilities, 11, 158

Disability, 39, 165, 213, 218, 243

Distance learning, 69

Dual-party relay services, 1

Early childhood, 148

Education, 195

Elderly, 47

Electronic publishing, 69

Electronic, 235

E-mail, 235

Emotionally disturbed, 87

Employee training, 69
Environment control, 321

Facsimile, 1, 203

Falls, 315

Gait and movement analysis, 321

Hard of hearing, 72, 173

Hearing devices, 47

Hearing impairment, 15

Home modifications, 283

Human computer interaction, 291

Hypermedia, 137

Hypertext, 137

IEP, 100

Impairments, 47

Inclusion, 77, 100

Instruction, 87

Integration, 148

Internet, 235

ISDN (integrated services digital network), 1

Job accommodation, 15

Learning disabilities, 129, 137

Least restrictive environment, 100

Legislation, 165, 244

Literacy, 117

Low back pain, 258

Low back research, 258

Low vision, 266

MainePOINT, 72

Mainstreaming, 307

Market research, 243

Mediated text, 137

Middle school, 129

Mildly disabled learners, 117

Mobility, 315

Multimedia, 117, 137

Network, 214

Noise (effects of), 39

Optical character recognition, 203

Organizational support, 158

Orthoses, 307

Paging systems, 69

Participatory action research, 62

Personal captioning, 26 
Physical disability, 321

Policy, 165, 244

Posturography, 315

Power wheelchairs, 307

Powered mobility, 321

Preschooler/disabilities, 148

Prostheses, 307

Prosthetics, 321

Qualitative research, 255

Quantitative research, 255

Rehabilitation, 235

Rehabilitation engineering, 258

Rehabilitation engineering research, 266

Rehabilitation (psychosocial aspects of), 62

Rehabilitation technology, 291

Relay service, 173

Research, 255

Robotics, 291

Safety/ear protection, 39

School change, 158

Scientific research, 255

Seating, 321

Sensory aids, 266

Service delivery (rural), 39

Severe disabilities, 77
Shared, 213

Sign language, 26

Social skills, 148

Social studies, 117

Special education, 100

Standard, 199, 218

Student with disability, 109

TDD, 199

Team, 109

Technology integration, 129

Technology, 11, 15, 77, 117, 137, 158, 173, 195

Telecommunications, $1,69,158,165,173,195$, 203, 213, 218, 235, 243

Telecommunications device for the deaf, 1

Telecourse, 72

Text Telephone, 1

Therapy, 87

TTY, 1

User interface, 218

Video compression, 203

Video transmission, 203

Worksite accommodation, 258

Worksite assessment, 258

Worldwide, 199 